\title{
Crystal Lattice Controlled SiGe Thermoelectric Materials with High Figure of Merit
}

\author{
Hyun Jung Kim ${ }^{1}$ Yeonjoon Park ${ }^{1}$ Glen C. King ${ }^{2}$ Kunik Lee $^{3}$ and Sang H. Choi ${ }^{2}$ \\ ${ }^{1}$ National Institute of Aerospace (NIA), Hampton, VA 23666, U.S.A. \\ ${ }^{2}$ NASA Langley Research Center, Hampton, VA 23669, U.S.A. \\ ${ }^{3}$ Federal Highway Administration of Department of Transportation, McLean, VA 22101, USA
}

\begin{abstract}
Direct energy conversion between thermal and electrical energy, based on thermoelectric (TE) effect, has the potential to recover waste heat and convert it to provide clean electric power. The energy conversion efficiency is related to the thermoelectric figure of merit ZT expressed as $\mathrm{ZT}=\mathrm{S}^{2} \sigma \mathrm{T} / \kappa$, T is temperature, $\mathrm{S}$ is the Seebeck coefficient, $\sigma$ is conductance and $\kappa$ is thermal conductivity. For a lower thermal conductivity $\kappa$ and high power factor $\left(\mathrm{S}^{2} \sigma\right)$, our current strategy is the development of rhombohedrally strained single crystalline SiGe materials that are highly [111]oriented twinned. The development of a SiGe "twin lattice structure (TLS)" plays a key role in phonon scattering. The TLS increases the electrical conductivity and decreases thermal conductivity due to phonon scattering at stacking faults generated from the $60^{\circ}$ rotated primary twin structure. To develop high performance materials, the substrate temperature, chamber working pressure, and DC sputtering power are controlled for the aligned growth production of SiGe layer and TLS on a $c$ plane sapphire. Additionally, a new elevated temperature thermoelectric characterization system, that measures the thermal diffusivity and Seebeck effect nondestructively, was developed. The material properties were characterized at various temperatures and optimized process conditions were experimentally determined. The present paper encompasses the technical discussions toward the development of thermoelectric materials and the measurement techniques.
\end{abstract}

\section{INTRODUCTION}

Increasing global interest in energy and environmental concerns has drawn attention to research in renewable and sustainable energy systems. Thermoelectric (TE) devices are promising because of their potential application as replacements or supplements to conventional energy conversion systems, such as waste heat recovery and environmentally-friendly refrigeration. ${ }^{1,2}$ Significant enhancement in the energy conversion efficiency of TE materials has been possible with morphologically nanostructured materials with low thermal conductivities..$^{3-5,9-12}$ The efficiency of TE materials is determined by the dimensionless Figure of Merit, ZT, expressed as $\mathrm{ZT}=\mathrm{S}^{2} \sigma \mathrm{T} / \kappa$, wherein the Seebeck coefficient $S(\mu \mathrm{V} / \mathrm{K})$, electrical conductivity $\sigma(\mathrm{S} / \mathrm{m})$, thermal conductivity $\kappa(\mathrm{W} / \mathrm{m} \cdot \mathrm{K})$, absolute temperature $\mathrm{T}(\mathrm{K})$ are the characteristic parameters and $\mathrm{Z}$ is multiplied by $\mathrm{T}$ to afford the Figure of Merit (FoM). ${ }^{2,13}$ Therefore, good TE materials require a high Seebeck coefficient, high electrical conductivity, and low thermal conductivity. Such materials are difficult to find in nature, and harder to engineer the individual properties without affecting other characteristics. SiGe based thermoelectric materials have been explored for over a decade as one of the most effective energy conversion materials at elevated temperatures. For applications at temperatures around $1000^{\circ} \mathrm{C}$, such as radio-isotope thermoelectric generators (RTGs) used on previous NASA space missions, SiGe alloys are among the known options. ${ }^{2}$ Here, thin film SiGe, including superlattice $\mathrm{SiGe}^{3-7}$ and twin lattice $\mathrm{SiGe}^{8}$, have shown promising trends in performance from their ability to scatter phonons at stacking faults created by the $60^{\circ}$ rotated primary twin structure. There exists a fundamental governing relationship in the rhombohedral epitaxy, i.e. the growth of cubic materials in $<111>$ orientation on top of the basal $c$-plane of the trigonal Sapphire crystals. The main 
idea of this new epitaxial growth method is to align $<111>$ direction of cubic diamond structure along with $<0001>$ direction of trigonal sapphire substrate. Often, the epitaxial layer affords two possible in-plane alignments shown in Figure 1b, i.e. the top-view.
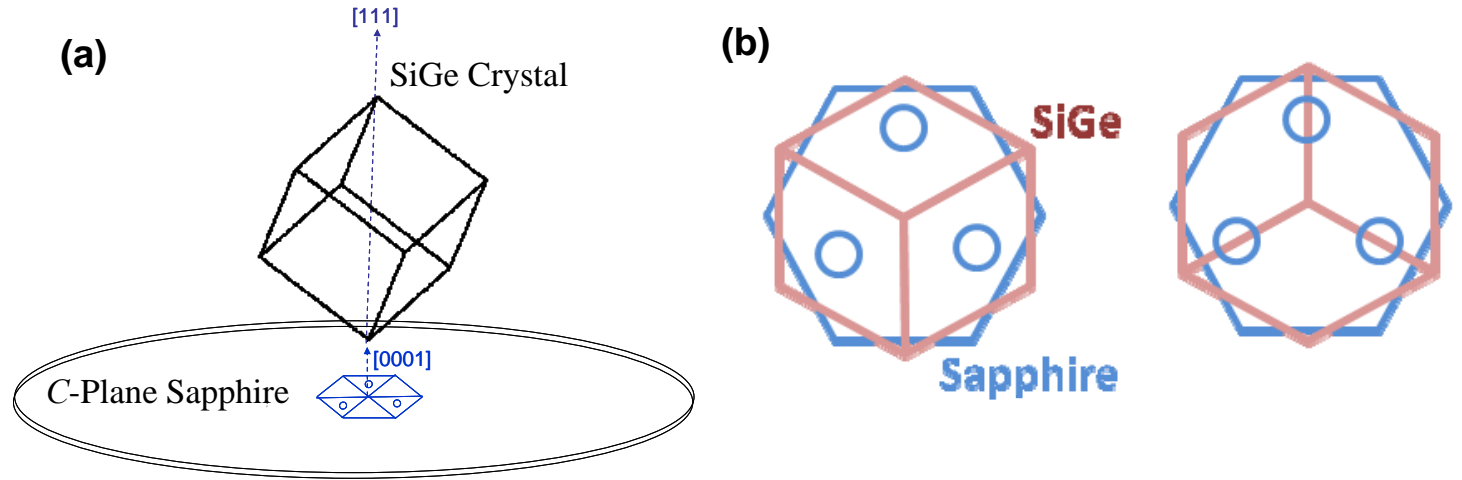

Figure 1. (a) Rhombohedral alignment: $<111>$ direction of cubic crystal is aligned with $<0001>$ direction of trigonal substrate, and (b) two possible in-plane azimuthal alignments inside rhombohedral alignment.

The two cubic crystals in Figure 1 (b) are coexisting twin crystals with one rotated by $60^{\circ}$ in $\{111\}$ plane. Therefore, if one cubic structure is a desired one, the other becomes the twin defect. By controlling single and twin structures of SiGe a film could be grown with a desired morphology.

Our current strategy to achieve a high FoM was to (1) increase electric conductivity through the development of rhombohedrally strained single crystalline SiGe materials and (2) grow highly [111]-oriented twinned SiGe material for decreased thermal conductivity. The verification of these results required a refined elevated-temperature measurement technique to verify the thermoelectric properties. A new thermoelectric characterization system was developed to measure all three parameters $(\mathrm{S}, \sigma$ and $\kappa$ ) nondestructively in the high-temperature range.

\section{EXPERIMENTAL}

1. Procedure

In this study, DC- and RF-sputtering methods were used for the growth of a SiGe layer on a 2-inch $c$-plane sapphire wafer. The wafer was cleaned with acetone, isopropanol, and deionized water before being transferred to a vacuum chamber and baked, using infrared heat, at $200^{\circ} \mathrm{C}$ for 1 hour. The chamber temperature was raised to $1000^{\circ} \mathrm{C}$ for a short time to remove any volatile contaminants (VOCs). The temperature of the chamber was then decreased to $820^{\circ} \mathrm{C}$ (thermocouple), and identical DC power was applied to the Si and Ge target materials. The growth of the first SiGe layer on the sapphire was then deposited by sputtering. Carbon coatings were applied to the back sides of the second and third series of sapphire wafers prior to SiGe sputtering to achieve higher substrate temperature by the absorption of infrared radiation. The growth temperatures of the second and third samples were $850^{\circ} \mathrm{C}$ and $890^{\circ} \mathrm{C}$, respectively. The variation of deposition conditions were tabulated and shown in Table I. While DC sputtering method was employed for the deposition of $\mathrm{Si}$ and $\mathrm{Ge}$, an RF-sputtering gun was used to co-sputter and mix boron into the SiGe layer as p-type dopant.

Table I. Deposition condition of SiGe films on $c$-plane sapphire substrate

\begin{tabular}{|c|c|c|c|c|c|}
\hline Sample & $\begin{array}{c}\text { Growth } \\
\text { temperature } \\
\left({ }^{\circ} \mathrm{C}\right)\end{array}$ & $\begin{array}{c}\text { Chamber } \\
\text { working pressure } \\
\text { (mTorr) }\end{array}$ & $\begin{array}{c}\text { High-purity } \\
\text { Ar gas } \\
(\mathrm{sccm})\end{array}$ & $\begin{array}{c}\text { DC sputtering } \\
\text { power } \\
(\mathrm{W})\end{array}$ & $\begin{array}{c}\text { RF sputtering } \\
\text { power } \\
(\mathrm{W})\end{array}$ \\
\hline $1^{\mathrm{st}}$ & 820 & 10 & 7 & 150 & $1-3$ \\
\hline
\end{tabular}




\begin{tabular}{|c|c|c|c|c|c|}
\hline $2^{\text {nd }}$ & 850 & 10 & 7 & 200 & $1-3$ \\
\hline $3^{\text {rd }}$ & 890 & 5.66 & 4 & 200 & $1-3$ \\
\hline
\end{tabular}

2. Sample Holder Clamp design

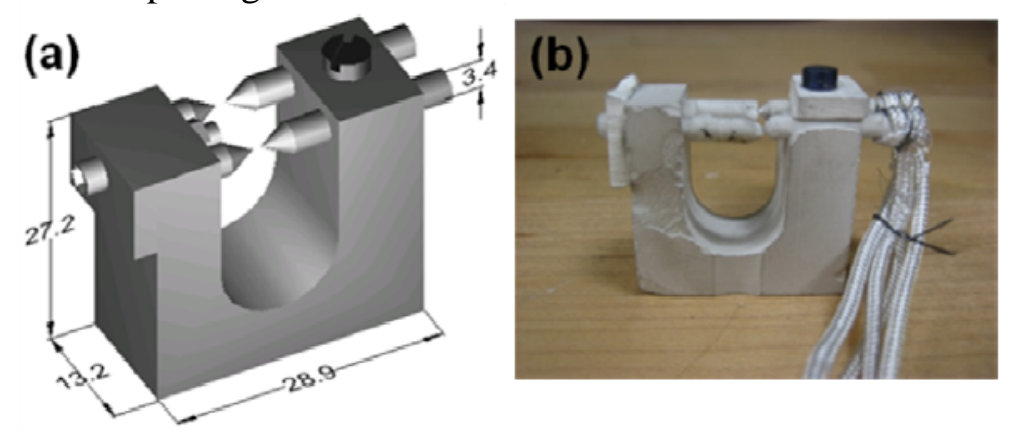

Figure 2. Hand-built sample holder for thermoelectric properties measurement (a) CAD drawing with dimensions in [mm] (b) actual item

Figure 2 shows the images of a sample holder that has been made out of a machinable ceramic tile (model \#960-15, Cotronics Corp.) along with other ceramic parts. Molybdenum screws (model \#4-40, Thermo Shield) were used due to a lower thermal coefficient of expansion (CTE) compared to alumina-based ceramics. ${ }^{14,15}$. One side had two S-type thermocouples made of 0.003 inch diameter wires of pure platinum and platinum/rhodium (10\%) (Alfa Aesar Inc.). The opposite side had two ceramic rods to hold a sample in its place. A small ceramic plate was screwed onto the sample holder to provide pressure to the rear ends of thermocouples. The wafer was inserted into the clamp and placed in the vacuum chamber. The chamber was then evacuated down to $1 \mathrm{E}-5$ torr, and argon gas was injected twice to purge any oxygen remaining in the chamber. Seebeck coefficient and electrical conductivity were measured from room temperature to $1000^{\circ} \mathrm{C}$ in $200^{\circ} \mathrm{C}$ increments.

\section{RESULTS AND DISCUSSION}

The growth parameters of SiGe material epitaxy on the $c$-plane of sapphire were the substrate temperature, the chamber working pressure, and the DC sputtering plasma power. The resulting orientation-dependent XRD results of the first and second samples are presented in Figure 3 where (1-a) and (2-a) show $\theta-2 \theta$ scans of the first and second samples in the perpendicular direction to the surface. The peaks (i) to (v) correspond to SiGe (111), sapphire (0006), SiGe (113), sapphire (00012), and SiGe (220), respectively. The strong SiGe (111) peak indicates that the majority of the SiGe layer was grown in [111] orientation on the substrate. The analysis of XRD peaks from the SiGe (111) show that all the samples have composition of $\mathrm{Si}_{0.15} \mathrm{Ge}_{0.85}$ due to the higher sputtering yield of Ge. ${ }^{16}$ The majority peaks are noted as (i) in graph (1-b) and (ii) in graph (2-b). The minority primary-twin crystal peaks rotated by $60^{\circ}$ are noted as (ii) in (1-b) and (i) in (2-b). The ratios are $60: 40$ and 0.3:99.7 in (1-b) and (2-b), respectively. For the samples grown at $820^{\circ} \mathrm{C}$, the $\{220\}$ peaks of the minority-twin crystal are always aligned with the $\{10-14\}$ peaks of sapphire. For the second sample grown at $850^{\circ} \mathrm{C}$, this tendency is reversed. The cubic shape diagrams in the plot for the second sample signify each peak with specific in-plane orientation. The $60^{\circ}$ difference between the two majority-twin SiGe layers, grown at different temperatures, indicates that there exists a temperature-dependent formation energy difference between majority-twin crystalline SiGe and minority-twin crystalline SiGe on the substrate. This also indicates that a variety of SiGe structures could be grown by regulating the deposition conditions. 

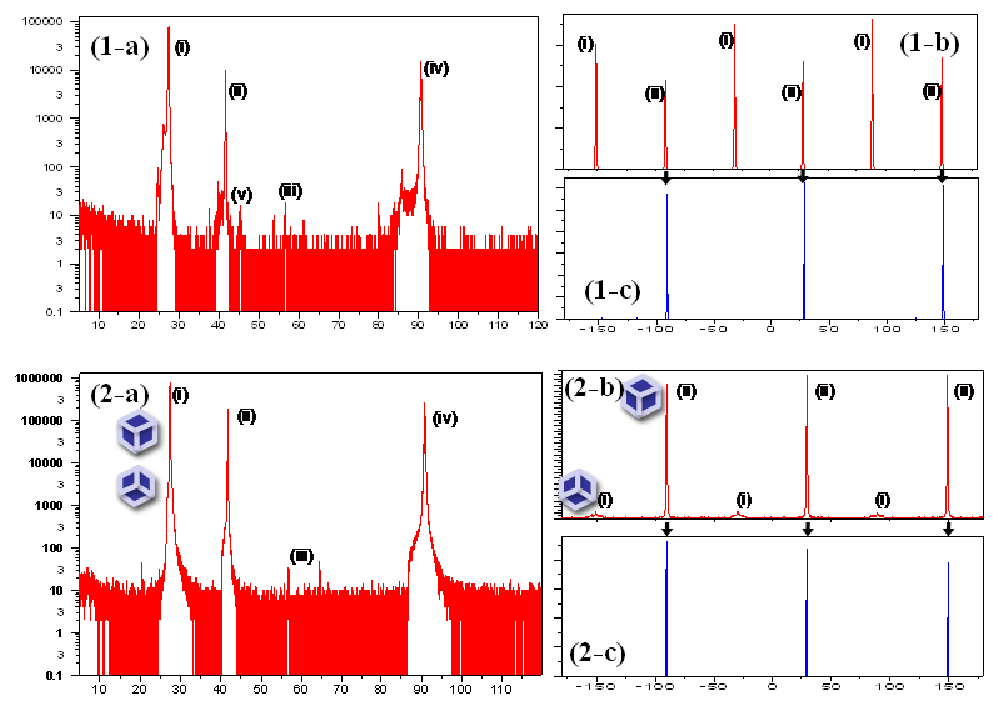

Figure 3. X-ray diffraction measurement on two-samples- (1) grown at $820^{\circ} \mathrm{C}$ and (2) $850^{\circ} \mathrm{C}$. Plot (a) shows $\theta-2 \theta$ scan in the direction normal to the surface, graph (b) shows phi scan of SiGe $\{220\}$ peaks, and graph (c) shows phi scan of sapphire $\{10-14\}$ peaks for the relative atomic alignment.

Graph (1-b) is in linear scale and graph (2-b) is in square root scale to show tiny twin peaks.

Figure 4 (a) shows the $\theta-2 \theta$ scans of the third sample in the direction normal to the surface and Fig. 4 (c) shows the wafer, a 99.9\% single-crystalline with mirror-like surface, seen from reflections and the camera. Single crystalline and twin defect maps are obtained as shown in (d) and (e). Figures (c) and (d) show that almost complete single-crystalline SiGe layer is fabricated on the basal plane of trigonal sapphire. Only a small amount of twin defect is formed on the edge of the wafer, since the deposition on the rim of the wafer was blocked by the wafer substrate holder. The third sample, sputter-deposited at $890^{\circ} \mathrm{C}$, has better single-crystalline characteristics compared to the second sample sputter-deposited at $850^{\circ} \mathrm{C}$. The high resolution transmission electron microscopy (HRTEM) image and its fast Fourier transform (FFT) of the interface mapping of $\mathrm{SiGe} / \mathrm{Al}_{2} \mathrm{O}_{3}$ (sapphire) of the third sample is shown in Figure 4(b) . This FFT result shows that a SiGe layer on the $\mathrm{Al}_{2} \mathrm{O}_{3}$ substrate has single crystalline structure. The zone axes are $\mathrm{Al}_{2} \mathrm{O}_{3}<01-10>$ and $\mathrm{SiGe}<-$ $112>$, respectively. The orientation relationships between the $\mathrm{Al}_{2} \mathrm{O}_{3}$ substrate and the SiGe single crystal layer are (0001) $\mathrm{Al}_{2} \mathrm{O}_{3} \|$ (111)SiGe and [01-10] $\mathrm{Al}_{2} \mathrm{O}_{3} \|$ [-112]SiGe. These results demonstrate that the [111]-oriented rhombohedral hetero-structure epitaxy of a cubic single crystalline SiGe layer on trigonal $c$-plane sapphire has been achieved. 
(a)

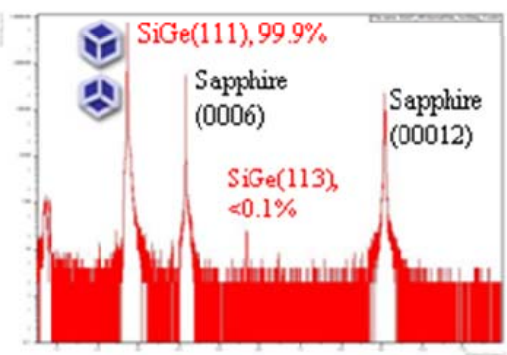

(c)

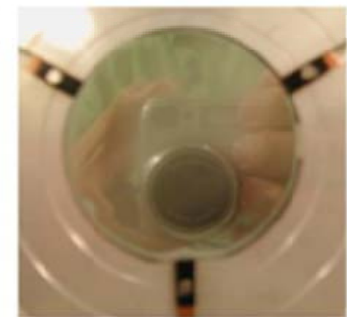

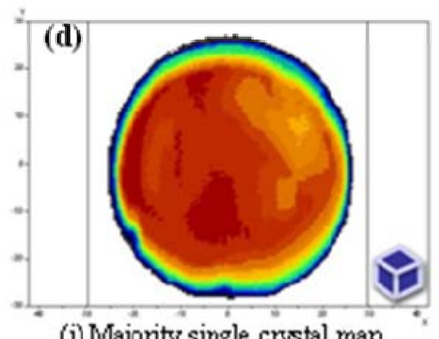

(i) Majority single crystal map
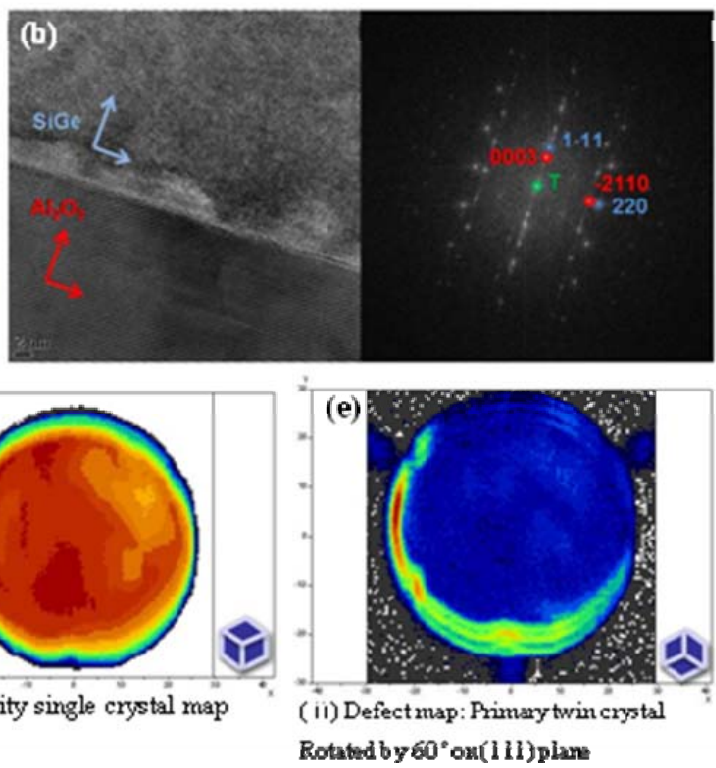

Figure 4. Analysis of the third SiGe sample. (a) shows $\theta-2 \theta$ scan in the direction normal to the surface, (b) HRTEM and its FFT result of the SiGe/sapphire interface, (c) photograph of the SiGe layer with mirror-like surface, (d) $X$ - $Y$ wafer mapping with majority $\{440\}$ peak marked with (i) shows distribution of a single crystal, (d) $X$ - $Y$ wafer mapping with minority $\{440\}$ peak, marked with

(ii) shows distribution of defect twin crystal.

Among the three thermoelectric property measurements, thermal conductivity can be obtained by several existing commercial systems that exploit the laser flash technique, transient method, or steady state method. ${ }^{14}$ However, for simultaneous thermoelectric conductivity and Seebeck coefficient measurements, not many systems have been devised as the difficulty in making firm electrical contact to the samples. Typically, for nondestructive measurement of thermoelectric properties, a probe station that utilizes mechanical preloading of thermocouples and electrodes to hold theses probes on the sample surface is used. This type of system requires a certain amount of force to sustain thermal and electrical contact without good electrical contact, voltage measurements fluctuate and cause significant error. It is difficult to mount the sample, thermal probes, and electrodes correctly, especially in the environment with elevated temperatures. Here, the physical properties of the sample holder materials are significantly altered by heat inducing changes in the contact area and could result in disconnections at the interface. Since there was no available device that measures all three parameters simultaneously at high temperatures, a system that measured the thermal conductivity at room temperature was modified to allow for Seebek coefficient and electrical conductivity measurements at elevated temperatures up to $1000^{\circ} \mathrm{C}$. A sample holder that keeps good mechanical contact at elevated temperatures up to $\sim 1000^{\circ} \mathrm{C}$, was developed that uses specially alloy screws as adjustable contact pressure points. 


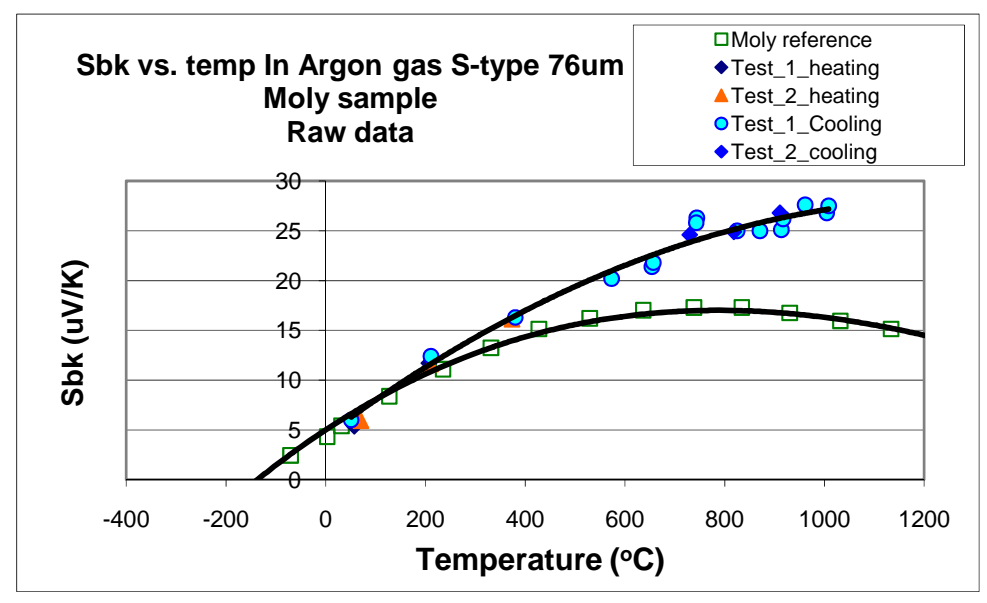

Figure 5. Seebeck coefficient measurement of a molybdenum sheet. Data points in green square are reference from CRC Handbook of Thermoelectrics. Data points in blue circles are raw data.

The data points plotted in green squares in Figure 5 are the tabulated values of the Seebeck coefficient of molybdenum used, as referenced in the CRC Handbook of Thermoelectrics, and circles in blue are raw data measured from this setup. It is apparent that there is a noticeable deviation of the raw data from the reference data. At this moment, the source of the error is unknown. A possibility is that the errors in the measurement might arise from the use of different junction materials at the thermocouple tip and the cables. To reduce such errors, the same materials constructing the probe lines and probes must be used. By introducing a correction factor, the deviation of measured data from the known values can be adjusted. The correction factor was found to be:

$$
y_{c o r}=-3.945 E-9 \cdot x^{3}+1.1039 E-5 \cdot x^{2}+0.00428 \cdot x-0.5843
$$

This correction factor (1) was applied to Seebeck coefficient measurements of nickel and tungsten. After applying the correction factor (1), the experimental results closely matched the referenced data.

A new setup for thermoelectric measurement was made for SiGe samples. The Seebeck coefficient and electrical conductivity are presented in Figure 6.

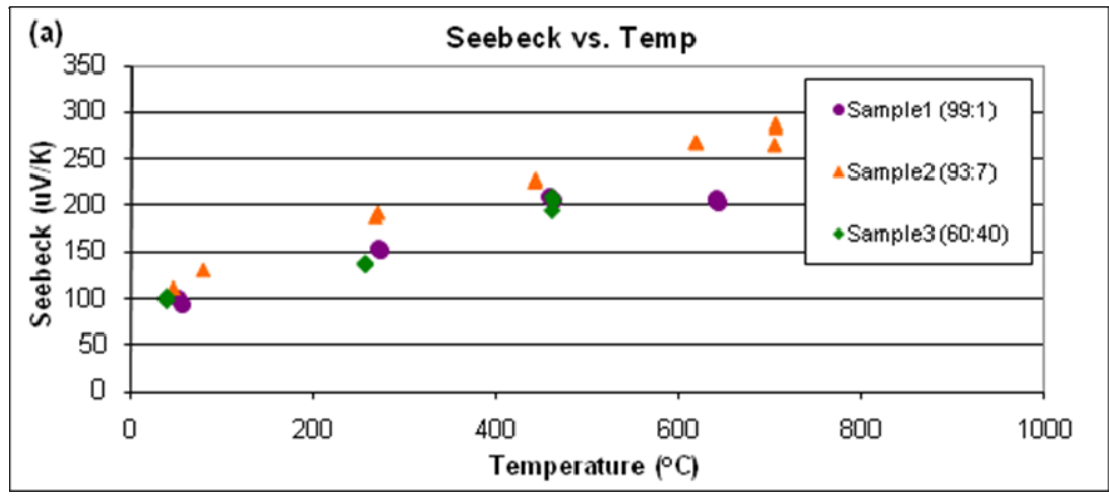




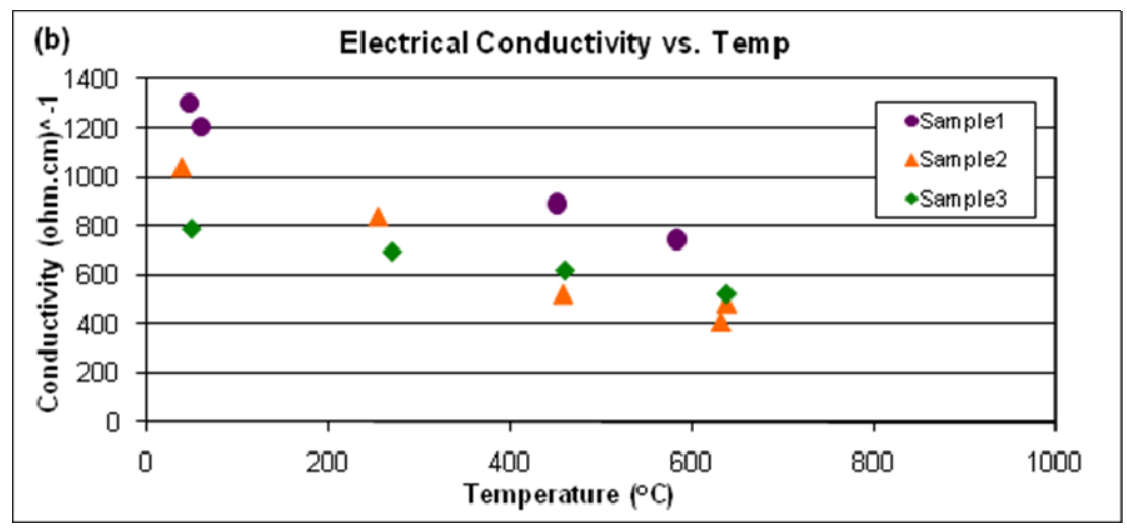

Figure 6. (a) Seebeck coefficient and (b) electrical conductivity of SiGe samples. The ratios of majority (single or twinned crystal structure) peak and minority (twinned or single crystal structure) peak are 99:1 in sample 1, 93:7 in sample 2, and 60:40 in sample 3, respectively.

Figures 6(a) and 6(b) show the post corrected Seebeck coefficient plot and the measured electrical conductivity of various SiGe samples with twinned phase, respectively. These values match closely with the data tabulated in the CRC Handbook of Thermoelectrics. ${ }^{2}$ The current experiment was carried out with two-point probe measurement followed by calibration through a four-point probe measurement done at room temperature to minimize measurement error. In the future, electrical conductivity will be measured by four-point probe method where the voltage drop is measured with two probes and current with the other two. The four-probe method is independent of contact resistance. However, unstable contact between the probes and sample could cause error in the electrical conductivity measurement. Contact resistance can be reduced and stabilized when pressure is applied between the probes and sample. At room temperature, a conductive paste is used to secure the force pressing the probes onto the sample. Such a paste cannot work at high temperature. Hence, good electrical conductivity and Seebeck coefficient measurements depend on how well the pressure is maintained at various temperatures. The relationship between thermal conductivity and thermal diffusivity are mathematically expressed as:

$$
\alpha=\frac{\kappa}{\rho \cdot c_{p}}
$$

Where $\alpha$ is the thermal diffusivity, $\kappa$ is the thermal conductivity, $\rho$ is the density of the material, and $c_{p}$ is the heat capacity. In this setup, thermal diffusivity of a SiGe sample was measured as shown in Figure 7. Thermal diffusivity and thermal conductivity of SiGe layers, on a sapphire substrate, were hard to measure because the SiGe layer was attached to the sapphire which has very high thermal conductivity. Therefore, a post-processing to remove the sapphire wafer was required for the evaluation of the characteristics. 


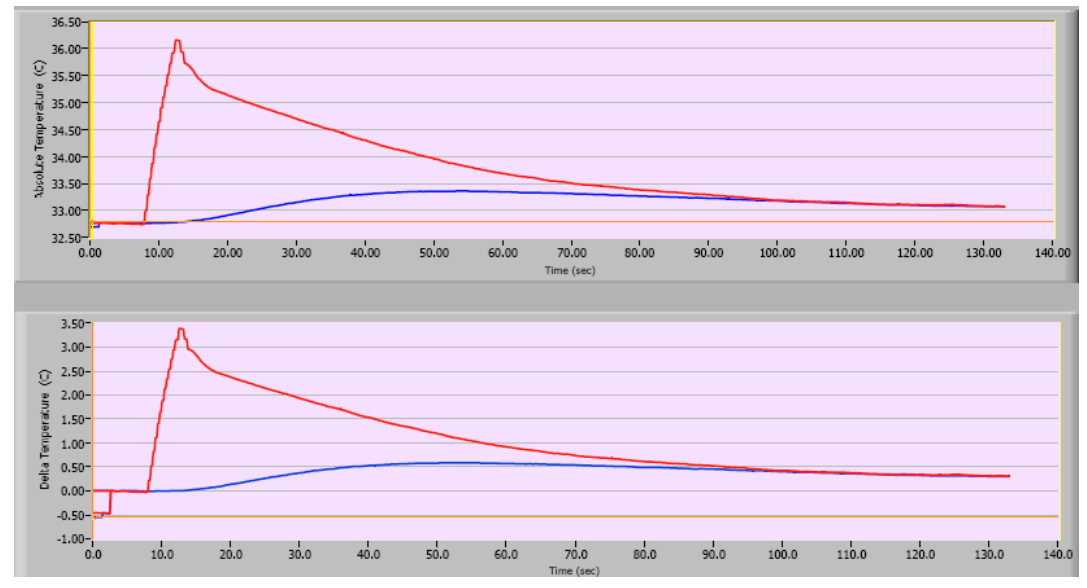

Figure 7. Thermal diffusivity measurement of SiGe sample. Temperature variation along time measured from the thermocouples near the heat source (red line, hot junction) and the heat sink (blue line, cold junction).

Unlike thermal conductivity measurement which requires the exact amount of heat entering and leaving the sample to be known, thermal diffusivity could be found by measuring the temperature of two locations and the time delay of temperature risings due to the heat transfer between these locations. Several attempts were made to measure all three parameters simultaneously at elevated temperatures, but only Seebeck coefficient and electrical conductivity were measured along the temperature excursions. Thermal diffusivity was not properly measured due to various problems including the contact between thermocouple tip and sample, the thickness of the sapphire wafer and SiGe layer, etc. In the future, state-of-the-art characteristics of the fabricated SiGe samples will be determined by first measuring the thermal conductivity separately and then, if required, developing a system that simultaneously measures all three parameters, required for full in-situ characterization.

\section{CONCLUSIONS}

Conventional SiGe materials have ZT values of 0.9 and 0.5 at $1200 \mathrm{~K}$ for n-type and p-type materials, respectively. Using our SiGe growth approach, ZT value can be improved. A highly [111]-oriented $\mathrm{Si}_{0.15} \mathrm{Ge}_{0.85}$ layer on the $c$-plane sapphire with a nearly single-crystalline phase and twinned phase has been fabricated. The large difference in the formation energy of the two crystals on the sapphire substrate depends on the substrate temperature. Rhombohedrally strained single crystalline SiGe increases electrical conductivity. Low thermal conductivity can be achieved through changing growth conditions, which in turn controls the density of stacking faults created by the $60^{\circ}$ rotated primary twin for phonon scattering. High electrical conductivity and low thermal conductivity lead to a higher figure of merit of thermoelectric materials. By controlling single and twin structures of SiGe through sputtering method, lattice-matched or twin-lattice structures were grown. There is no standard method or reference material for Seebeck coefficient. Although techniques for measuring electrical conductivity have been well established, measurement will not be reliable unless good Ohmic contact is ensured. Contact also matters for Seebeck characterization, which requires voltage measurement. This served as a motivation to develop a device to measure all three parameters simultaneously by ensuring good contact between the probe and sample at very high temperature. In the present report, a thin film measurement system that measures Seebeck 
coefficient and electrical conductivity at temperature up to $1000^{\circ} \mathrm{C}$ has been demonstrated. Thermal diffusivity measurement using this system has been demonstrated in room temperature range, presenting the possibility of developing thermal diffusivity measurements in elevated temperature range as well. A custom-built sample holder will be used to measure SiGe films of various morphologies at high temperatures in the future.

\section{ACKNOWLEDGMENTS}

This work was supported by the Creativity and Innovation Program at NASA Langley Research Center. The authors appreciate the assistance of Dr. Jaewoo Kim at NIA, Hampton, VA for SEM EDX data, Dr. Shiho Iwanaga at Caltech with thermoelectric measurement system, Dr. Intae Bae at State University of New York at Binghamton for TEM data, and Mr. Hyung Bin Bae, a TEM researcher at KAIST, South Korea.

\section{REFERENCES}

1. G. S. Nolas, J. Sharp, and H. J. Goldsmid, “Thermoelectrics: basic principles and new materials developments” (Springer, Berlin; New York, 2001)

2. D. M. Rowe, “CRC handbook of thermoelectric” (CRC Press, Boca Raton, FL, 1995)

3. T. C. Harman, P. J. Taylor, M. P. Walsh, and B. E. LaGorge, Science 297, 2229 (2002)

4. R. Venkatasubramanian, E. Silvola, T. Colpitts, and B. O’Quinn, Nature 413, 597 (2001)

5. G. Chen, Phys. Rev. B 57, 14958 (1998)

6. S. -M Lee, D. G. Cahill, and R. Venkatasubramanian, Appl. Phys. Lett. 70, 2957 (1997)

7. B. Yang, W. L. Liu, K. L. Wang, and G. Chen, Appl. Phys. Lett. 81, 3588 (2002)

8. Y. Park, Glen C. King, and Sang. H. Choi, J. Cryst. Growth 310, 2724 (2008)

9. M. S. Dresselhaus, G. Chen, M. Y. Tang, R. G. Yang, H. Lee, D. Wang, Z. Ren, J. P. Fleurial, and P. Gogna, Adv. Mater. 19, 1043-1053 (2007)

10. L. D. Hicks and M. S. Dresselhaus, Phys. Rev. B 47, 12727 (1993)

11. K. F. Hsu, S. Loo, F. Guo, W. Chen, J. S. Dyck, C. Uher, T. Hogan, E. K. Polychroniadis, and M. G. Kanatzidis, Science 303, 818-821 (2004)

12. A. I. Hochbaum, R. Chen, R. D. Delgado, W. Liang, E. C. Granett, M. Naharian, A. Majumdar, and P. Yang, Nature 451, 164 (2007)

13. T. M. Tritt, Ed., "Semiconductors and Semimetals,” San Diego (2001)

14. F. C. NIX, and D. MACNAIR, Phys. Rev. 61, 74 (1942)

15. P. Hidnert, and H. S. Krider, J. Res. Nat. Bur. Stand. 48, 3, 209 (1952)

16. D. L. Smith, “Thin-Film Deposition-Principles and Practice,” Academic Press, New York, 1995 (Appendix) 